\title{
聚乙撑亚胺衍生物中不等性 微区的核磁共振研究
}

黄启斌 双云度 黄增绪

(北京药物化学研究所, 北京 102205)

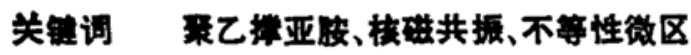

合成聚合物模拟酶, 特别是模拟水解酶, 最主要的问题是阐明环境对接在高分子上的功能 基影响的各种机制. 已知酶促反应中活性基团相当大的反应性, 很大程度上取决于基团周围 微区的作用 ${ }^{[1]}$. 因此, 在合成聚合物模型中, 设计能够影响基团活性的类似于酶的微区对于理 解酶的特殊催化能力是至关重要的.

微区在天然酶的研究中是一个普遍采用的概念 ${ }^{[2]}$. 因此在功能模拟酶中,引人、发展并表 征这一概念对于酶模型的设计具有重要意义. 现已知道生物体系的高级结构就是由亲水性微 区和疏水性微区形成的微观非均一结构决定的. 亲水性与疏水性、结晶性与非结晶性, 刚性与 柔性等组合形成的馆段或接枝共聚物在固、液相中会产生微观区域分离. 特别地, 亲疏并存 一极性的亲水性微区与非极性的疏水性微区镶湠交错的聚合物也许是最接近天然酶的理想 模型。

改性的聚乙撑亚胺 (PEI) 衍生物被广泛地用于模拟酶的研究 ${ }^{[3,4}$. 关于 PEI 上非极性接 枝基团的区域环境, Klotz 等人 ${ }^{[5,6]}$ 利用氟标记的核磁共振 (NMR) 技术和电子自旋共振 (ESR) 方法进行了初步研究, 结果表明, 这类聚合物中存在不同性质的区域。本文利用高分 辨 ${ }^{1} \mathrm{H}$ NMR 技术,用一个不含生色团的小分子探针一乙醇研究了聚乙撑亚胺系列衍生物的 区域结构, 首次观察到不等性微区的存在.

\section{1 材料与方法}

\section{1 材料}

PEI 行生物的合成及表征 ${ }^{1)}$, 其结构通式如 Scheme 1. 详细的结构参数由 ${ }^{1} \mathrm{H}$ NMR 谱 确定, 见表 1 .

\section{2 方法}

本文的 ${ }^{1} \mathrm{H} N \mathrm{NM}$ 谱实验于 JEOL GX400 型超导傅里叶变换 NMR 仪上进行. ${ }^{1} \mathrm{H} N M R$ 工作频率为 $399.65 \mathrm{MHz}$. 采用 $\varnothing 5 \mathrm{~mm}$ 标准 NMR 样品管, 以気代水作溶剂. 样品浓度约 为 $20 \mathrm{mg} / 0.5 \mathrm{ml}$. 实验在室温下进行.

对聚合物 PEI-C ${ }_{16}-\mathrm{OXPh}$ 的 ${ }^{1} \mathrm{H}$ NMR 实验, 谱宽为 $5000 \mathrm{~Hz}$, 数据点 16384 , 脉冲宽度

1993-01-06 收稿, 1993-05-25 收修改稿.

1) 黄启斌㴓士学位论文, 北京, 1992. 


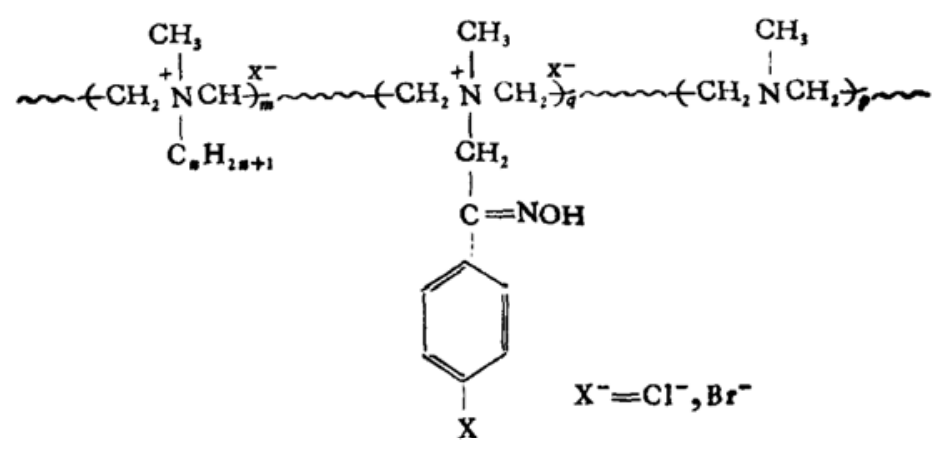

图解 1

为 $3.5 \mu \mathrm{s}$, 弛豫延迟 $1 \mathrm{~s}$, 累加 16 次. 其它聚合物的 'H NMR 实验与上述条件类似.

\section{2 结果与讨论}

图 1 显示了在进行 PEI- $\mathrm{C}_{16}-\mathrm{OXPh}$ 的结构测定时意外获得的图谱. 在 $\delta 3.40-3.80 \mathrm{ppm}$ 间,我们得到了一组“孪生峰”, 高场峰出现在 $\delta 3.55 \mathrm{ppm}$, 低场峰出现在 $\delta 3.62 \mathrm{ppm}$ 附近. 这 对孪生峰究竟是什么质子引起的共振峰呢? 我们考察了 $\mathrm{PEI}-\mathrm{C}_{16}-\mathrm{OXPh}$ 整个合成过程中可能 引人的杂质分子, 最大可能是不同环境中乙醇分子的亚甲基被甲基分裂产生的共振峰. 因为 (a) 在合成 PEI- $\mathrm{C}_{16}-\mathrm{OXPh}$ 过程中可能引入的其它杂质,如甲醇、乙醚、吡啶、甲醛、甲酸均不 能在此区域产生四重峰, 并且实验表明这些杂质已完全除尽;（b）碳谱结果表明存在乙醇杂 质;（c）聚合物主链分子及十六烷基的部分均不存在这样的多重峰, 并且大分子骨架上的质 子难以产生如此清晰可辨的共振峰，而小分子则可以产生这样的峰；（d）二维 ${ }^{\mathrm{t}} \mathrm{H}^{-}{ }^{-} \mathrm{HNMR}$ $\operatorname{cosY}$ 实验表明该孪生峰主要是由乙醇的甲基上的质子分裂的 ${ }^{1)}$. 显然, 确认这对孪生峰均是 乙醇亚甲基的质子峰是合理的.

然而, 乙醇分子为什么会产生如此的孪生峰呢? 我们猜想乙融分子一定是进人了 PEI$\mathrm{C}_{16}-\mathrm{OXPh}$ 溶液中不同的微区, 质子的低场共振主要是在亲水微区, 在高场质子共振则肯定是 在不同于水的环境中, 即疏水微区. 当孪生峰出现的, 乙醇分子在这两种微区的父换达到平 衡, 或者说乙醇分子在疏水微区已达饱和. 为了验证这一猜想, 在测定 $\mathrm{PE} 1-\mathrm{C}_{16}-\mathrm{OXPh}$ 时, 我 们额外地加入 1 滴 (约 $0.05 \mathrm{ml}$ ) 无水乙醇到样品管中, 结果发现高场共振几乎不变, 而低场 共振则增加很多 (图 2), 显然这意味着乙醇的量已达饱和, 而额外加人的乙醇则主要分布在水 中(低场). 由于乙醇分子是极性分子, 它能进人疏水微区, 说明由 $\mathrm{PEI}-\mathrm{C}_{16}-\mathrm{OXPh}$ 形成的疏 水微区并非完全是非极性的, 至少在微区的周围具有一定的弱极性, 即不等性.

为了进一步验证不等性微区的存在, 本文还考察了 PEI 系列衍生物在有、无乙醇存在下 的 ${ }^{1} \mathrm{H}$ NMR 谱(表 1). 结果表明 (a) 不具有长链烷基结构的 PEI-OXH、PEi-OXMe 和 PEI- $\mathrm{C}_{8}-\mathrm{OXH}$ 体系不显示孪生峰, 说明聚合物胶束结构对疏水微区的形成起一定的作用. 而 PEI-OXN 体系中, 乙醇的孪生峰可能是由阳离子聚㿝及苯环的特殊效应造成的 ${ }^{1}$; (b) 样 品处理时, 千燥彻底的 $\mathrm{PEI}-\mathrm{C}_{12}-\mathrm{OXCl}$ 和 $\mathrm{PEI}-\mathrm{C}_{16}-\mathrm{OXCl}$ 体系, 没有发现孪生峰; (c) 对于 PEI- $\mathrm{C}_{12}-\mathrm{OXH}$ 和 PEI- $\mathrm{C}_{16}-\mathrm{OXMe}$ 体系, 乙醇杂质倾向于分布在亲水微区; (d) 对于 PEI$\mathrm{C}_{16}-\mathrm{OXN}$ 体系, 在一定条件下, 少量的乙醇杂质可以只在疏水微区; (e) 不同聚合度的聚合 物, 如 PEI- $\mathrm{C}_{16}-\mathrm{OXN}$, 对乙醇的质子的化学位移影响不大, 这说明不同聚合度的聚合物可以形 成相似的疏水微区;（f）当乙醇在疏水微区和亲水微区达到平衡后, 额外乙醇只沚、入亲水微 


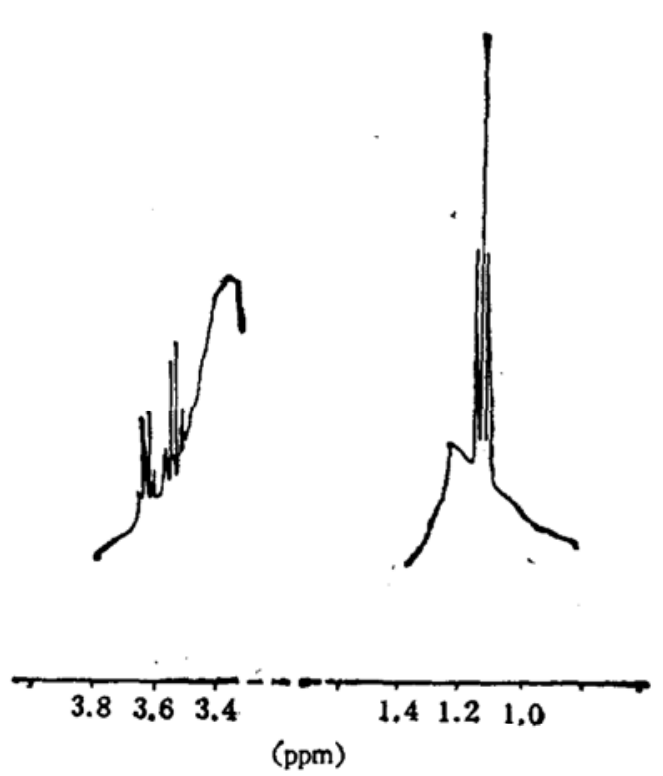

图 1 探针分子乙䤃的孪生峰

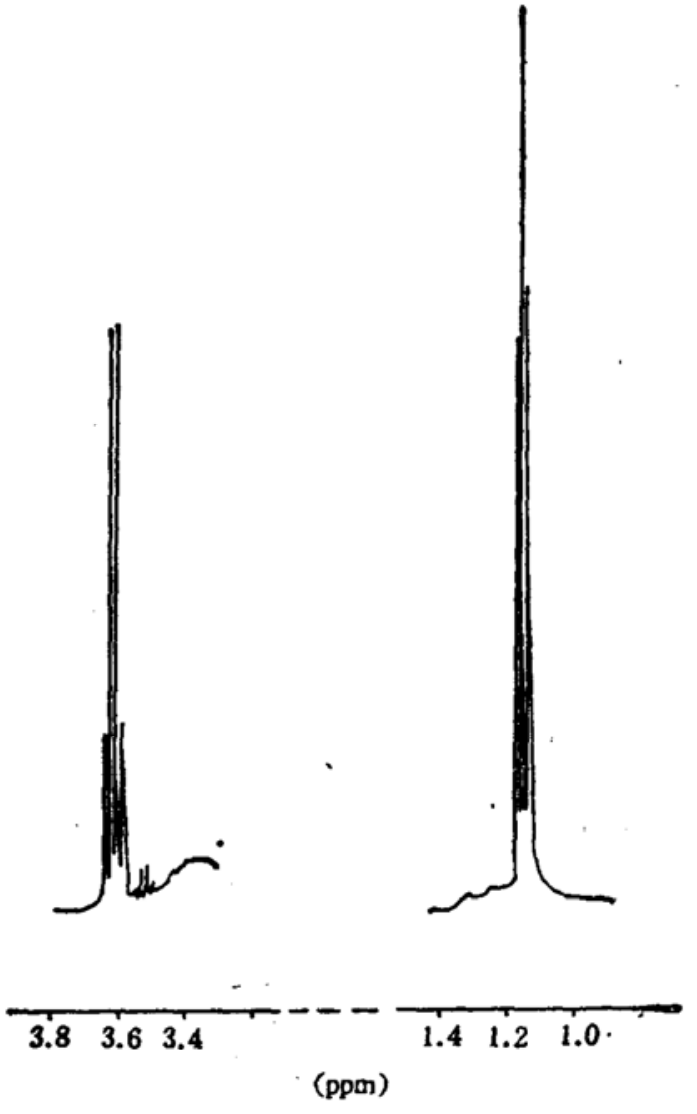

图 2 过量乙醇的孪生峰

表 1 聚合物溶液中乙醇质子的化学位移")

\begin{tabular}{|c|c|c|c|c|c|c|c|}
\hline 体系 & $\mathbf{x}$ & $n$ & $m$ & $q$ & $p$ & $\mathrm{CH}_{3}(\mathrm{ppm})$ & $\mathrm{CH}_{2}(\mathrm{ppm})$ \\
\hline $\left.\mathrm{CC}_{4}^{\mathrm{b}}\right)$ & & & & & & 1.17 & 3.58 \\
\hline PEI-OXH & $\mathrm{H}$ & 0 & 0 & 14 & 86 & $1.20(w)$ & - \\
\hline PEI OXN & $\mathrm{NO}_{2}$ & 0 & & & & 1.16 & $3.55,3.62(w)$ \\
\hline PEI-OXMe & OMe & 0 & 0 & 6 & 94 & $1.15(w)$ & - \\
\hline $\mathrm{PEI}-\mathrm{C}_{2}-\mathrm{OXH}$ & $\mathrm{H}$ & 8 & 5 & 19 & 76 & - & - \\
\hline PEI-C ${ }_{12}-\mathrm{OXH}$ & $\mathrm{H}$ & 12 & 7 & 25 & 68 & 1.19 & 3.65 \\
\hline \multirow[t]{2}{*}{ PEI- $\mathrm{C}_{16}-\mathrm{OXH}$} & $\mathrm{H}$ & 16 & 4 & 6 & 90 & 1.19 & $3.57,3.65$ \\
\hline & $\mathrm{H}$ & 16 & 12 & 55 & 33 & 1.22 & $3.60,3.70$ \\
\hline PEI $-\mathrm{C}_{12}-\mathrm{OXCl}$ & $\mathrm{Cl}$ & 12 & 4 & 7 & 89 & - & $\ldots$ \\
\hline $\mathrm{PEI}-\mathrm{C}_{16}-\mathrm{OXCl}$ & $\mathrm{Cl}$ & 16 & 10 & 23 & 67 & - & - \\
\hline PEI-C ${ }_{16}-\mathrm{OXMe}$ & $\mathrm{OMe}$ & 16 & 9 & 28 & 63 & $1.20(w)$ & $3.65(w)$ \\
\hline $\mathrm{PEI}-\mathrm{C}_{16}-\mathrm{OXBr}$ & $\mathrm{Br}$ & 16 & 4 & 6 & 90 & 1.19 & $3.58,3.65$ \\
\hline \multirow[t]{3}{*}{ PEI- $C_{16}-O X N$} & $\mathrm{NO}_{2}$ & 16 & 6 & 13 & 81 & 1.20 & $3.60,3.70$ \\
\hline & $\mathrm{NO}_{2}$ & 16 & 9 & 27 & 64 & 1.19 & $3.57 .-$ \\
\hline & $\mathrm{NO}_{2}$ & 16 & 10 & 11 & 79 & 1.20 & $3.58,3.68$ \\
\hline PEI- $\mathrm{C}_{16}-\mathrm{OXPh}$ & $\mathrm{Ph}$ & 16 & 4 & 6 & 90 & 1.16 & $3.55,3,62$ \\
\hline $\mathrm{PEI}-\mathrm{C}_{16}-\mathrm{OXPh}$ & $\mathrm{Ph}$ & 16 & 4 & 6 & 90 & 1.14 & $3.55,3.62(s)$ \\
\hline$+\mathrm{CH}_{3} \mathrm{CH}_{2} \odot \mathrm{H}$ & & & & & & & \\
\hline
\end{tabular}

a) s-D强峰, w-D强峰; b) 摘自 Sadtler 光谱图集.

区, 几乎不进入疏水微区, 如在 $\mathrm{PEI}-\mathrm{C}_{16}-\mathrm{OXPh}$ 中.

表 1 的数据还说明聚合物体系只影响乙醇的 $\mathrm{CH}_{2}$ 的质子化学位移,并产生孪生峰, 而对 
$\mathrm{CH}_{3}$ 质子的化学位移并不产生较大影响, 且没有孪生峰产生, 这表明 $\mathrm{CH}_{2}$ 具有不同的环境, 而 $\mathrm{CH}_{3}$ 却在相似的环境中. 因此,我们设想了醇分子的 $\mathrm{CH}_{3}$ 位于亲水区, 而 $-\mathrm{CH}_{2} \mathrm{OH}$ 部分

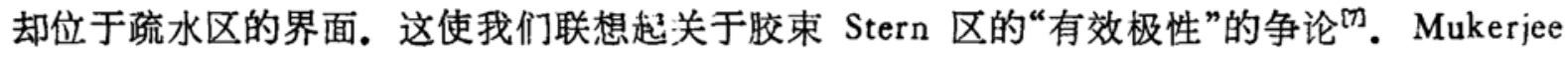
指出胶束的 Stern 的有效极性与甲醇相似，而 Sudholter 以 1-甲基-十二烷基吡啶碘形成的 胶束作为本征微观介质的极性研究了有关双电层最内层的信息, 认为内层的极性与乙醇相当, 并指出 Stern 层有相当的均匀性. 本文的结果表明, 疏水区的界面是有极性的, 至少与乙醇 的极性相当. 另一尚无定论的问题是象水、乙醇这样的极性分子究竟能否穿过胶束：本文的 实验表明极性的乙醇分子是可以渗透到疏水微区(胶束)中去的, 但究竟能否达到真正的胶束 的核心则难以肯定. 从高场的化学位移来看, 在 $\mathrm{PEI}-\mathrm{C}_{16}-\mathrm{OXPh}$ 疏水微区中的乙醇分子中的 亚甲基的化学位移几乎与在完全极性溶剂 $\left(\mathrm{CCl}_{4}\right)$ 中的相同,这是否意味着乙醇分子进人了非 极性介质的内核呢? 还是意味着疏水区也有一定的极性? 这些问题尚待进一步研究.

致谢哀心感谢军事医学科学院仪器中心缪振春老师在核磁共振谱测定和解释时给予 的无私帮助.

\section{考文献}

[1] Page, M. I., Jencks, W. P., Proc. Natl. Acad. Sci. USA, 1971, 68:1678.

[2] Kirsh, Yu. E., Kabanov, V. A., Eur. Polym. J.. 1974, (10):393.

[3] Koltz, 1. M., Ann. N. Y. Sci., 1985, 434(v).

[4] 黄启陚、邓云度,科学通报，1992，37(23): 1798 .

[5] Klotz, I. M., Macromolecules, 1984, 17:198.

[6] Klotz, I. M., Johnson, T. W., Macromolecules, 1974, 7:618.

[7] Fendler, J. H., Membrence Mimetic Chemistry, John Wiley \& Sons, New York, 1982, 30 -38. 